\title{
MULTI-LAYER RECOVERY ENABLED WITH END-TO-END SIGNALING
}

\author{
D. Verchere ${ }^{1}$, D.Leclerc, A.Noury, B.Ronot, M.Vigoureux, O.Audouin, A.Jourdan \\ D. Papadimitriou², B.Rousseau, G. Luyts \\ S. Brockmann ${ }^{3}$ W. Köber, G. Eilenberger \\ ${ }^{1}$ Alcatel R\&I, Route de Nozay, 91460 Marcoussis, France,Dominique.Verchere@alcatel.com \\ ${ }^{2}$ Alcatel Bell, Alcatel Bell, Francis Wellesplein 1, B-2018 Antwerpen, Belgium, \\ ${ }^{3}$ Alcatel SEL, Holderaeckerstrasse 35, 70499 Stuttgart, Germany
}

Abstract: Within GMPLS framework, the signaling protocol Resource reSerVation Protocol with Traffic Engineering extensions (RSVP-TE) is extended to support the requirements of an Automated Switched Optical Network architecture. This paper presents the extensions of the end-to-end connection services in an overlay network built on two control planes. RSVP-TE protocol extensions are first described between an IP/MPLS router and a SDH/GMPLS core optical cross-connect, defining GMPLS-UNI. Dimensioning of three scenarios proving the benefits of GMPLS-UNI is discussed.

\section{INTRODUCTION}

Recently IETF has been made a lot of progress in explaining how its GMPLS protocol suite satisfies the requirements of Automated Switching Optical (ASON) Network architecture specified by ITU-T. In one hand the GMPLS framework specifies all the protocol capabilities in terms of signaling (e.g. RSVP-TE), routing (e.g. OSPF-TE) and link management (e.g. with LMP). And in the other hand, ITU-T ASON recommendations specify the architecture and requirements for the Automatic Switched Transport Network as applicable to SDH transport networks.

GMPLS generalizes the label switching concepts introduced in MPLS to all switching technologies. GMPLS extends the label switching capabilities to 
network elements hosting non-packet based switching matrix, from labeled packet, frame, cell switching technologies to circuit switching technologies including SDH and Optical Transport Hierarchy (OTH).

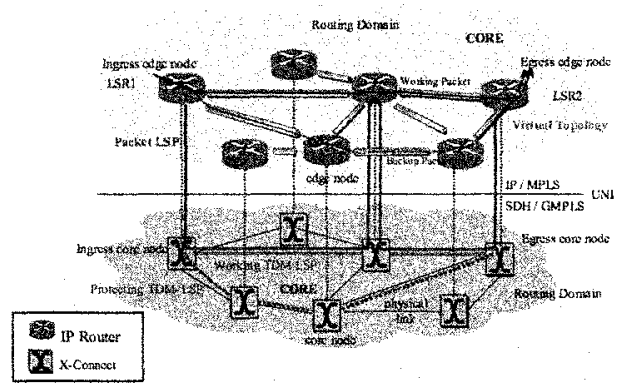

Figure 1. Multi-Layer network architecture: IP/MPLS over SONET-SDH/GMPLS

E.g. an IP/MPLS-over-SDH/GMPLS network architecture is considered within this paper, all the results presented apply to an SONET/GMPLS transport network. The core network comprises two control planes: IP packet transport layer on top of the time-division-multiplexing (TDM) transport layer. The TDM layer consists of optical cross-connects (OXCs) interconnected by one or more optical fibers (physical links). Each SDH connection corresponds to a logical link for the IP/MPLS layer. These links interconnecting Label Switching Routers (LSRs), constitute a virtual topology. The control plane interactions i.e. the functional coordination capabilities between the layers are described in the following section. The section 2 provides the new signaling extensions required at the router-OXC interface to enable end-to-end connection coordination. In section 3, three multilayer network recovery scenarios are presented and the section 4 allows to position the new User-to-Network Interface GMPLS-UNI capabilities.

\section{CONTROL PLANE INTERCONNECTION MODELS}

As defined in [1], different control plane interconnection configurations can be deployed at the User-to-Network Interface (UNI) reference point. Optical Internetworking Forum did specify the OIF UNI version 1.0 implementation agreement [2]. This signaling interface is applicable when the IP/MPLS client and SDH/GMPLS server belong to separate administrative domains. End-to-end signaling functions, the exchange of reach-ability, topology or explicit route connection information between the control plane instances are not possible. The messages exchanged through the OIF UNI are limited to requests for and acknowledgements of the establishment and tear-down of transport connections. 
OIF UNI introduces GENERALIZED_UNI object enabling address separation, both types and spaces increasing complexity. More GENERALIZED_UNI object functionality can be addressed with EXPLICIT_ROUTE object.

From the OIF-UNI inherent complexity, a user-to-network signaling interface is being developed at the IETF: the GMPLS-UNI (a.k.a. GMPLS for overlay networks [3]). The major differences between the OIF UNI and the GMPLS UNI signaling interface can be summarized as follows: (i) simplify end-reference point identification to numbered (IPv4/IPv6) or unnumbered interfaces (ii) allow clientdriven explicit routing, typically loose routing and (iii) maintain a single end-toend RSVP[4] signaling session (see the more in [1]).

The GMPLS UNI interface [3] is defined as an RSVP-TE signaling interface for packet-over-circuit and circuit-over-circuit networks that provides: connection provisioning and multi-layer recovery. The former includes LSP establishment, deletion, modification, and status inquiry. The latter entails failure notification, establishment of resource disjoint paths in response to a failure.

For end-to-end recovery, GMPLS-UNI enables to associates explicitly one or more working connections with one protecting connection with the ASSOCIATION object [4]. This object is carried in the Path message of the working LSP and in the protecting LSP. Each transit node along the LSP route transmits the ASSOCIATION object without any modification. GMPLS UNI allows the usage of the PROTECTION object as extended in [4]. The client LSR's explicitly specify the desired end-to-end LSP recovery level starting at the ingress and terminating at the egress router. The protection types defined are: dedicated protection, protection with extra-traffic, (pre-planned) re-routing without extratraffic and dynamic re-routing. The Notify message provides a fast mechanism to notify non-adjacent nodes of events such as LSP failures [4]. Notify messages are only generated when explicitly configured during LSP establishment with the NOTIFY_REQUEST object. The Notify message does not have to follow the same path as the Path/Resv messages used to establish the LSP and is not processed at intermediate nodes. From GMPLS-UNI signaling function such as (i) end-to-end explicit routing and label control, (ii) end-to-end LSP association, (iii) end-to-end LSP protection, (iv) end-to-end route diversity, and (v) multi-layer fast notification, three recovery scenarios are developed.

\section{MULTI-LAYER NETWORK RECOVERY SCENARIO}

To dimension the recovery in IP/MPLS-over-SDH networks, three scenarios have been developed. For each, the OIF-UNI and the GMPLS-UNI interfaces are compared. The Italian network topology was used, composed with 30 point-ofpresences (PoP's) interconnected with 62 physical links. At each PoP, a single 
backbone router office is set-up. The line side interfaces of the router are supposed to be the same as OXC ones i.e. STM-X with $X=1,16$ or 64 . The total number of STM-X ports is summed from add/drop ports and in/out ports. Through each scenario, the interface STM-X sends/receives VC-4-Xc virtual containers.
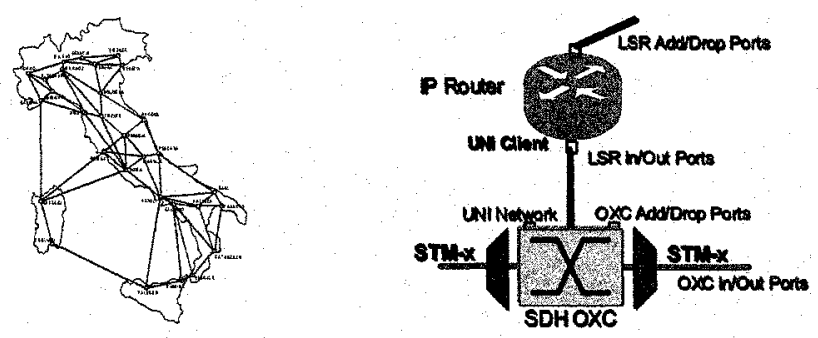

Figure 2. Network Topology (left), a single backbone router office architecture (right)

To allow packet LSP to be provisioned, TDM LSP are triggered following a bottom-up sequence.

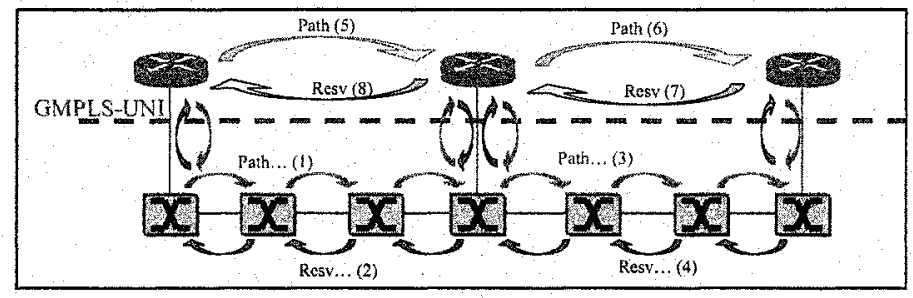

Figure 3. Packet LSP over TDM LSP multi-hop provisioning

In the scenario 1 (figure 4), a packet LSP is established between router $B$ and F across the virtual topology. For the TE link between $B$ and $F$, there exits different route from edge $\mathrm{OXC} b$ to $f$. The working SDH connection carries the traffic from $\mathrm{B}$ outgoing interface to $\mathrm{F}$ ingoing interface. The recovery connection is established between the edge $\mathrm{OXC} b$ to $f$ and is route diverse from working connection. Each recovery connection can protects several connections.

The TDM recovery connections are pre-planned (see [1]) i.e. network resources are computed, selected and reserved but not cross-connected. The recovery resources can not carry any traffic because they are not provisioned at the data plane but only at the control plane level (i.e. "soft-provisioned"). Soft-provisioned connections enable the sharing of the recovery resources. In case of failure, switching from the working to the recovery sub-connection occurs at the edge i.e. $\mathrm{OXCs} b$ and $f$. The issue intrinsic to scenario 1 is at UNI reference point between the router and its adjacent $\mathrm{OXC}$, it requires link protection. The protection only works between the edge cross-connects inside the transport network. The recovery 
resources can not transport router driven best-effort traffic because the recovery switching point is located inside SDH/GMPLS network.

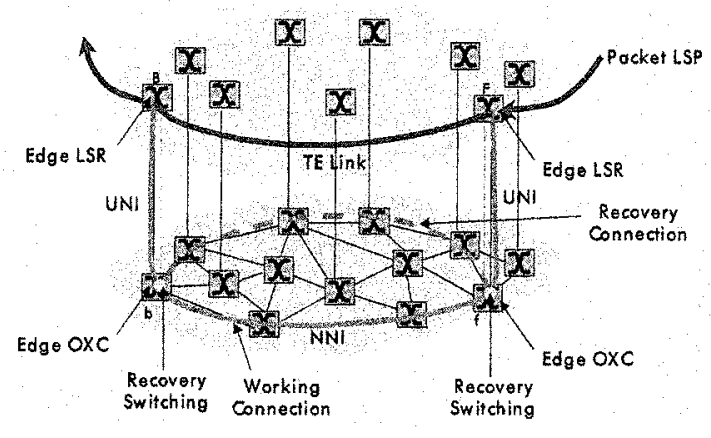

Figure 4. Scenario 1: SONET/SDH sub-connection recovery

The second recovery scenario (figure 5) has its recovery switching point at the edge router interface. The connection provisioning and recovery switching are both processed at the TDM switching granularity. During connection establishments, the router subscribes to be notified for link failures. The working SDH connection carries the traffic from B outgoing Packet-over-SDH (PoS) interface until the F ingoing PoS interface.

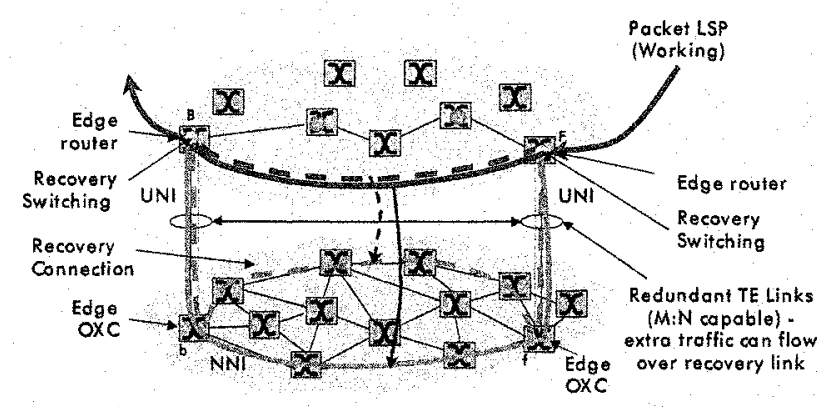

Figure 5. Scenario 2: end-to-end SONET/SDH recovery connection.

The recovery connection is established from $\mathrm{B}$ to $\mathrm{F}$ and is route diverse. The protecting TDM connections are soft-provisioned and can be shared between several source-destination pairs. For a link failure event, after reception of Notify message, the ingress router signals the corresponding soft-provisioned recovery connection. This bottom-up end-to-end connection recovery requires a coordination based on the Notify message from the OXC to the router. This scenario requires to implement GMPLS-UNI between each router-OXC pair. Finally this scenario enables dual homing protection at UNI reference point i.e. the 
edge devices when an edge router is connected to two edge routers. In the scenario3 represented in fig. 6, the provisioning of the packet LSP's is completely processed on the logical topology created by the IP/MPLS network[5]. Each packet LSP and its detour LSP (i.e. its protecting LSP) can use one or more TE links.

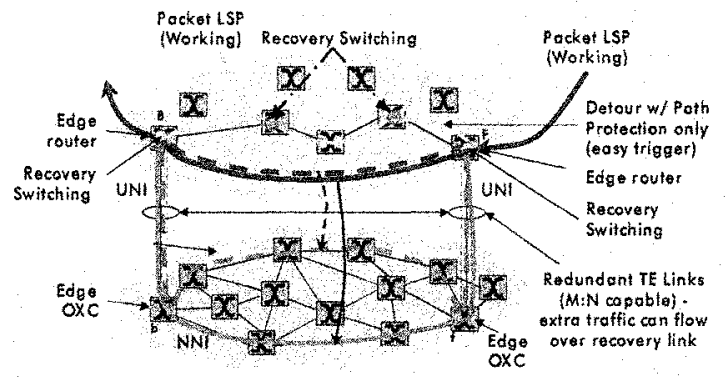

Figure 6. Scenario 3: Packet Label Switched Path Local Recovery

In the transport network, the recovery SONET/SDH connection used to protect the working SONET/SDH connection is used by the detour LSP protecting the packet LSP. This packet LSP is nested in the working TDM connection and its detour LSP is nested in the recovery TDM connection. In this scenario, the recovery SONET/SDH connection are fully reserved i.e. the network resource along the TDM recovery connection route are computed, selected and reserved and cross-connected (see [1] for details). Each detour LSP reserves the same amount of bandwidth than its attached protected packet LSP. A detour LSP can be shared between one or more packet LSP's i.e. the bandwidth reserved for the detour LSP can be shared by other detour LSP's. This technique is referred as "Facility backup" in [5]. In scenario 3, there is no dedicated TDM connection either for working connection or for recovery connection. Each TDM LSP can nest a set of packet LSP's and detour LSP's. Inside a TDM connection, the set of packet LSP's are route diverse from the other packet LSP's protected by the detour LSP's.

\section{GMPLS-UNI DIMENSIONINGS}

The results prove that the GMPLS-UNI based multi-layer recovery is superior in terms of recovery speed and/or resource utilization (Figs. 7-9). Fig.7 illustrates, the total number of interfaces i.e. router+OXC for STM-1 (left) and STM-16 (right) interfaces. Fig.8 gives STM-64 filling ratio with VC-4 (left) and VC-4-16c (right) and Figure 9, the traffic recovery ratio in function of the recovery delay (ms). For the same recovery level (IP/MPLS protection), sce. 2 is the optimal for resource usage and scenario 3 is the fastest. 

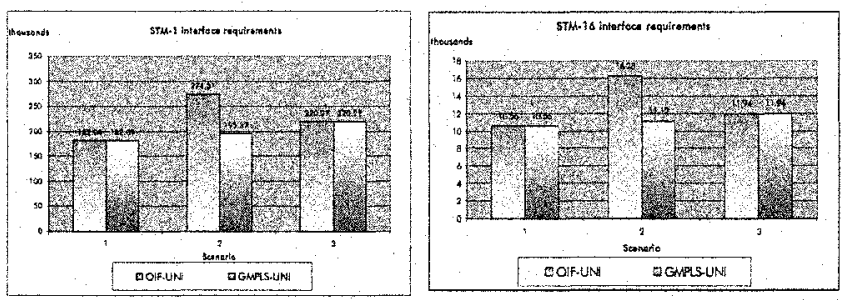

Figure 7. Number of router + OXC interfaces: STM-1 (left) \& STM-16 (right).

Figure 7 is obtained with an average of TE link connectivity degree 10.77 logical link interface per router (see fig.1). The requirements are provided depending of the LSR-OXC interface:OIF-UNI vs GMPLS-UNI. The difference between sce. 1 and 2 is due to the link interconnection at OXC-LSR, in sce.1 no interface protection is implemented. In sce.3, working packet LSP and its detour are routed over link diverse connections. Fig. 8 compares STM-64 filling ratio that is fraction of STM-64's bandwidth reserved to carry VC-4 or VC-4-16c granularities. For connectivity degree $4.13,7.43,10.77$ and 29.00 , average number of optical hops between the edge routers for $\mathrm{SDH}$ connections is $3,3.5,3.8$ and 5.1, resp. "3" corresponds to the connection explicit route: ingress router $\rightarrow$ ingress $\mathrm{OXC} \rightarrow$ egress OXC $\rightarrow$ egress router. In fig. 9, the recovery ratio (\% of traffic affected by failure \& recovered) is plotted. In sce. 3 , traffic recovery is processed by router. Recovery of connection translates into recovery of all working packet LSP's.
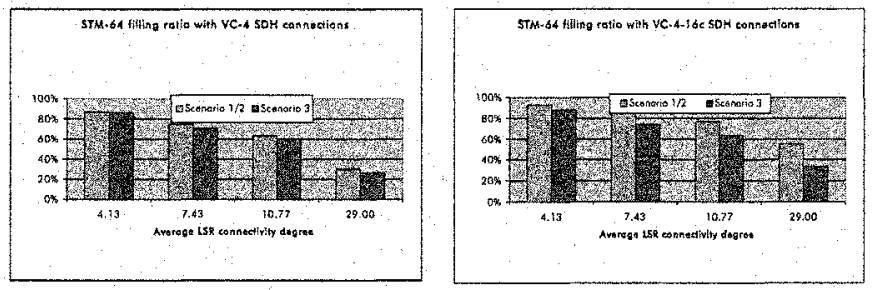

Figure 8. STM-64 filling ratio: VC-4 (left) / VC-4-16c (right) SDH connections

The recovery time is the duration elapsed from a failure detection and the time the failed connection is recovered, $T^{(i)}$. The recovery of the first connection is completed at $T_{0}^{(i)}(i=1,2,3)$, the recovery of the ultimate connection is at $T_{100}^{(i)}$. It appears that the recovery speed of SDH connection (scenario $1 \& 2$ ) is slower than packet LSP-based recovery, SDH/GMPLS recovery requires an additional round trip time to signal network resource compared to IP/MPLS one: $T_{0}^{(3)} \leq T_{0}^{(1)} \approx T_{0}^{(2)}$. Due to TDM soft-provisioning applied in sce. $1 \& 2$, protecting SDH connection requires to be cross-connected before traffic can be switched onto them. The difference between sce. 1 and $2(\sim 5 \mathrm{~ms})$ is due to one additional hop along the route 
of the Notify message. For scenario $1 \& 2$, IP/MPLS recovery mechanism can be a fallback mechanism of SDH/GMPLS recovery failure.

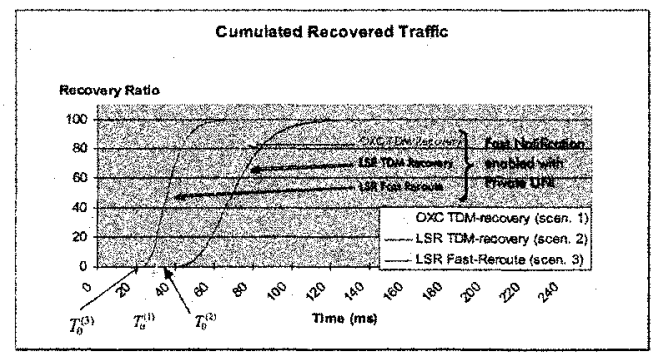

Figure 9. Traffic Recovery Ratio (\%) in function of the Recovery delay (ms)

These recovery mechanisms are resource optimal, provide fast recovery and yield a deterministic network status.

\section{CONCLUSIONS}

GMPLS UNI benefits were proved qualitatively and quantitatively facilitating sequences of recovery actions between layers which in turn allows optimizing the resource usage and the delays inferred by the restoration of the traffic. New contributions are further extending the capabilities of the GMPLS UNI by allowing routing information to be exchanged to evolve towards an augmented model. The GMPLS UNI as defined by the IETF, is an important step in the continued evolution towards the delivery of integrated and intelligent network solutions.

\section{REFERENCES}

[1] D. Papadimitriou, W. Körber, B. Rousseau, S. Brockmann, D. Verchère, "The Private User Network Interface", OSA-Journal. Optical. Network. Vol.3, No.3, pp119-132

[2] "User Network Interface (UNI) 1.0 Signaling Specification", OIF, October 2001.

[3] "GMPLS-UNI RSVP Support for the Overlay Model", G. Swallow et al., Internet Draft (work in progress), draft-ietf-ccamp-gmpls-overlay-04.txt, April 2004.

[4] "RSVP-TE Extensions in support of End-to-End GMPLS-based Recovery", J.P. Lang et al., Internet Draft, draft-ietf-ccamp-gmpls-recovery-e2e-signaling-01.txt, May 2004.

[5] "Fast Reroute Extensions to RSVP-TE for LSP Tunnels", P. Pan (Ed.) et al., Internet Draft (work in progress), draft-ietf-mpls-rsvp-lsp-fastreroute-02.txt, February 2003. 